\section{Screening for coeliac disease: what evidence is required before population programmes could be considered?}

\section{E H Young, N J Wareham}

\section{Commentary on the paper by Tommasini et al}

su the traditional doctor-patient consultation, the patient initiates a visit and the physician's imperative is to respond to the best of his or her ability. However, in a screening programme, the initiation comes from health professionals, and many commentators have argued that this poses different ethical challenges. ${ }^{1}$ In particular it is argued that the level of evidence concerning the balance of overall benefit and harm from the screening programme must be much greater than that for a traditional therapeutic intervention.

Although screening may intuitively be logical for a particular condition which is prevalent and detectable in the preclinical stage, intuition is not an appropriate basis for commencing a screening programme. A number of criteria have been described that allow the evidence for screening programmes to be more formally evaluated. ${ }^{2}$ Calls are regularly made for the introduction of population screening programmes, such as that for coeliac disease (CD) in the paper by Alberto et al in this issue. ${ }^{3}$ These screening criteria form the basis for evaluating the strength of the evidence supporting that call. They also highlight key uncertainties, which could be the subject of future research. This process has been undertaken for screening for type 2 diabetes in adults, ${ }^{4}$ and the history of the debate on this topic may shed some light on how to reach a more speedy decision for CD.

\section{THE DISEASE}

The effectiveness of a screening programme is closely related to the characteristics of the disease for which screening is proposed. CD is an important health problem as it is common, affecting 1 in 120-300 people in Europe, ${ }^{5}$ and its associated morbidity and excess mortality are well recognised. ${ }^{67}$ However, the natural history of asymptomatic $\mathrm{CD}$ is less well understood, although some patients with unrecognised disease have extra-intestinal manifestations such as growth restriction ${ }^{8}$ or associated autoimmune disease. ${ }^{6}$ The prevalence of associated autoimmune disorders in $\mathrm{CD}$ is related to the duration of exposure to gluten, ${ }^{9}$ providing a strong imperative for screening and early treatment.

\section{THE TEST}

As with type 2 diabetes screening, there is considerable debate around the relative merits of different screening modalities for CD. The performance of a potential screening test is dependent on diagnostic thresholds for the disease and the availability of a simple, precise, and validated test that is acceptable to the population. Serological tests are easy to administer and have an important role in screening for CD. A number of highly sensitive and specific markers are available. ${ }^{56}$ In this issue, Alberto and colleagues show the high positive predictive value of one autoantibody, serum anti-h-tTG-ab. ${ }^{3}$ For any screening test, there should be an agreed policy on the further diagnostic investigation of individuals with a positive test result. In this instance, individuals with a positive antibody result underwent the gold standard diagnostic test of small intestine biopsy. Screen negative children were not investigated further. However, without biopsying screen negative children the sensitivity or specificity of the

\section{THE TREATMENT}

A key issue in evaluating any screening test is to consider whether screening and subsequent treatment result in health gain. For patients with symptom detected $C D$, delay in diagnosis unfavourably affects patients' prognosis. Treatment with a gluten-free diet for life provides good relief of symptoms and is effective in reversing abnormal gut pathology. Prompt treatment also decreases mortality. ${ }^{7}$ For adults, quality of life is improved on a gluten-free diet. ${ }^{10}$ Children develop normally and report a quality of life similar to that of children in the general population. ${ }^{11}$ However, not all adults with CD attain the same degree of subjective health as the general population. ${ }^{12}$ Provided that patients with CD adhere to a strict diet, test cannot be established. the prognosis is excellent and there are no data to suggest that there is an excess mortality in this group.

Yet the benefits of early detection and treatment of screen detected asymptomatic CD have not been proved. With treatment, quality of life improves for those whose disease was screen detected..$^{13}$ The impact of treatment on mortality and morbidity for asymptomatic individuals is less clear. Asymptomatic CD is often associated with other pathology including reduced bone density, and iron and folate deficiencies, which if left untreated would account for a number of chronic health issues. Silent disease may also account for the worsening of other serious medical conditions, ${ }^{14}$ although this link is by no means established.

It is the evaluation of evidence concerning the costs and benefits of treatment for individuals with screen detected disease that is often lacking. One cannot necessarily translate results from treatment trials in people with established disease and apply the evidence to the population with screen detected, possibly asymptomatic, disease. In type 2 diabetes even though patients may believe their illness to be serious, many can reconcile such beliefs with poor adherence to medical advice about lifestyle. ${ }^{15}$ Many patients assume that the presence of symptoms is a necessary precursor of complications and equate freedom from symptoms with freedom from risk. This is highly relevant to $C D$, since screening would result in asymptomatic individuals being advised a lifelong gluten-free diet. Although the diet may be efficacious, its effectiveness in real life is a function of the degree to which patients adhere to advice.

\section{THE SCREENING PROGRAMME}

Population screening is always a major undertaking, and the merits of universal population screening must be weighed up against targeting high risk subgroups. The health benefits of mass screening for $\mathrm{CD}$ are not known, and in particular no good trial evidence exists to indicate that screening for CD would achieve significant health gain or that it would outweigh the disbenefits of screening. Ideally, evidence of effectiveness in reducing mortality and morbidity should come from randomised trials. A trial would also make it possible to calculate more accurately the costs of screening.

\section{IMPLICATIONS}

In the case of type 2 diabetes, the argument surrounding the benefits of screening has continued since the 1960s. Although the disease and the test criteria are easily met, as with CD the major uncertainties concern the benefits and disbenefits of early detection and treatment. 
It becomes increasingly difficult to undertake a randomised controlled trial of screening as evidence mounts, and there is probably a window of opportunity in which people are sufficiently uncertain to tolerate randomisation. The lesson for researchers into disorders such as $\mathrm{CD}$ is not to miss that window, otherwise the debate about whether or not to screen may run for decades.

\section{ACKNOWLEDGEMENTS}

EHY is an MRC Career Development Fellow.

Arch Dis Child 2004;89:499-501.

doi: 10.1136/adc.2003.046839

Authors' affiliations
E H Young, N J Wareham, MRC
Epidemiology Unit, Cambridge, UK

Correspondence to: Dr N J Wareham, MRC Epidemiology Unit, Strangeways Research
Laboratory, Worts Causeway, Cambridge CB1 8RN, UK; njw1004@medschl.cam.ac.uk

\section{REFERENCES}

1 Cochrane AL, Holland WW. Validation of screening procedures. Br Med Bull $1971 ; 27: 3-8$.

2 The UK National Screening Committee. Criteria for appraising the viability, effectiveness and appropriateness of a screening programme. www.nsc.nhs.uk/pdfs/criteria.pdf (accessed 16 December 2003).

3 Tommasini A, Not T, Kiren V, et al. Mass screening for coeliac disease using antihuman transglutaminase antibody assay. Arch Dis Child 2004:89:512-5.

4 Wareham NJ, Griffin SJ. Should we screen for type 2 diabetes? Evaluation against National Screening Committee criteria. BM 2001;322:986-8.

5 Farrell RJ, Kelly CP. Celiac sprue. N Engl J Med 2002;346:180-8

6 Green PHR, Jabri B. Coeliac disease. Lancet 2003;362:383-91.

7 Corrao G, Corazza GR, Bagnardi V, et al. Mortality in patients with coeliac disease and their relatives: a cohort study. Lancet 2001;358:356-61
8 Bingley PJ, Williams AJK, Norcross AJ, et al. Undiagnosed celiac disease at age seven: population based prospective birth cohort study. BMJ 2004;328:322-3.

9 Ventura A, Magazzu G, Greco L. Duration of exposure to gluten and risk for autoimmune disorders in patients with celiac disease. Gastroenterology 1999;117:297-303.

10 Green PHR, Stavropoulos SN, Panagi SG, et al. Characteristics of adult celiac disease in the USA results of a national survey. Am J Gastroenterol 2001;96:126-31.

11 Kolsteren MMP, Koopman HM, Schalekamp G, et al. Health-related quality of life in children with celiac disease. J Pediatr 2001;138:593-5.

12 Hallert C, Granno C, Grant C, et al. Quality of life of adult coeliac patients treated for 10 years. Scand J Gastroenterol 1998:33:933-8.

13 Mustalahti K, Lohiniemi S, Collin P, et al. Glutenfree diet and quality of life in patients with screendetected celiac disease. Eff Clin Pract 2002;5:105-13.

14 Kaukinen K, Halme L, Collin P, et al. Celiac disease in patients with severe liver disease: gluten-free diet may reverse hepatic failure. Gastroenterology 2002;122:881-8.

15 Murphy E, Kinmonth AL. No symptoms, no problem? Patients' understandings of noninsulin dependent diabetes. Fam Pract 1995; 12:184-92.

children in prison were not even covered by the provision of the Children Act 1989. Children could therefore be treated in prison in ways that would have triggered child protection investigations if they had occurred outside the penal system.

The recent Children's Green Paper Every child matters ${ }^{3}$ pledges a commitment by government to secure and protect the welfare of every child in England. Child protection is given a high profile and made a high priority for all agencies. Despite this the Home Office still proposes that the only factor to be considered when a court passes sentence on a child is stopping them reoffending. The welfare of a child does not appear to be a consideration. ${ }^{4}$

Gould and Payne rightly highlight the lack of systematic information about the health of this vulnerable group of children as an appropriate matter of concern to paediatricians and an area where more research and information is needed.

Children who get into trouble with the law and end up in prison are often the same group who suffer abuse, school exclusion, family disruption, and mental health problems. Many have been brought up in care and have special educational needs. They are the nation's most distressed and vulnerable children. There must be a fundamental change in the way society sees these troubled children.

They have complex and challenging needs. Their health, education, and welfare is sorely neglected. Prison is no place for them. They need looking after, educating, and protecting-not locking up. on some fronts-more willingness to
Reform won a judicial review against the Home Office in November 2002, listen to children, more help for young people leaving care, and a commitment there was also plenty of criticism. The excessive and increasing numbers of custody for lesser offer highlighted as was the low age of Scotland, 10 in England, and 13 in most ther European states. There was children are held in young offend a high level of violence, bullying, , and suicide. The report expres children of asylum seekers was Convention for children. Most of these committee's most controversial repeal the 142 year old law giving parents the right to use "reasonable chastisement" in disciplining their children. $^{2}$

Until the Howard League 
Arch Dis Child 2004;89:500-501. doi: 10.1136/adc.2003.043695

Correspondence to: Dr M Mather, Bexley Primary Care Trust, 221 Erith Road, Bexleyheath, Kent DA7 6HZ, UK; mary. mather@bexley.nhs.uk

\section{REFERENCES}

1 Gould J, Payne H. Health needs of children in prison. Arch Dis Child 2004;89:549-50.

2 Harvey R. Review of the concluding observations made by the United Nations Committee on the Rights of the Child in its examination of the UK's implementation of the United Nations Convention on the Rights of the Child 1989. childRIGHT 2002;190:3-5.

3 DfES. Every child matters. London: HMSO, 2003.

4 Home Office. Youth justice-the next steps. London: HMSO, 2003.

\section{IMAGES IN PAEDIATRICS}

\section{Periorificial dermatitis with dramatic response to zinc}

A 6 month old female child presented with vesicular and crusted lesions together with well defined, erythematous, glazed, moist plaques on perioral, perigenital, and perianal regions for last one month (fig 1). It was associated with intermittent diarrhoea. She was the first child of consanguineous parents and was normal at birth. She had been weaned from breast feeding over the past two and a half months.

Her anthropometric measurements were normal. Serum albumin was normal, but alkaline phosphatase (200 IU/l) and zinc levels $(20 \mu \mathrm{g} / \mathrm{dl})$ were low. The patient was diagnosed as having acrodermatitis enteropathica and was started on an oral preparation of zinc sulphate $(0.2 \mathrm{mg} / \mathrm{kg} /$ day $)$. There was marked improvement of all lesions within 48 hours and complete clearing by 7 days (fig 2). Continuation of maintenance oral zinc was advised.

Acrodermatitis enteropathica is a rare autosomal recessive disorder. There is a genetic defect of the synthesis, structure, or function of a zinc binding ligand

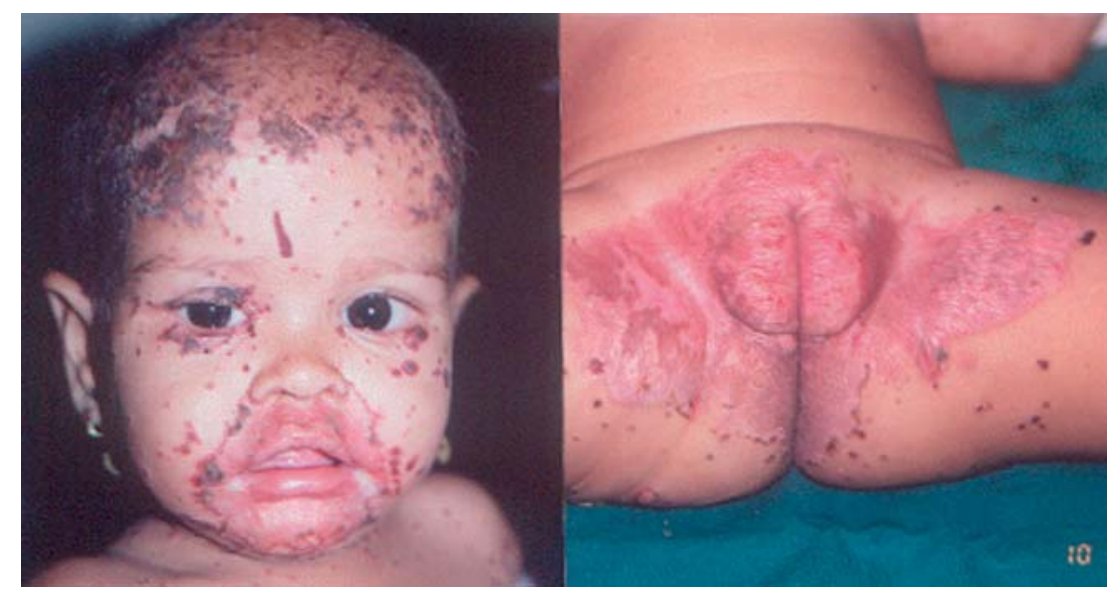

Figure 1 Dermatitic lesions and erythematous, glazy plaque on periorificial areas.

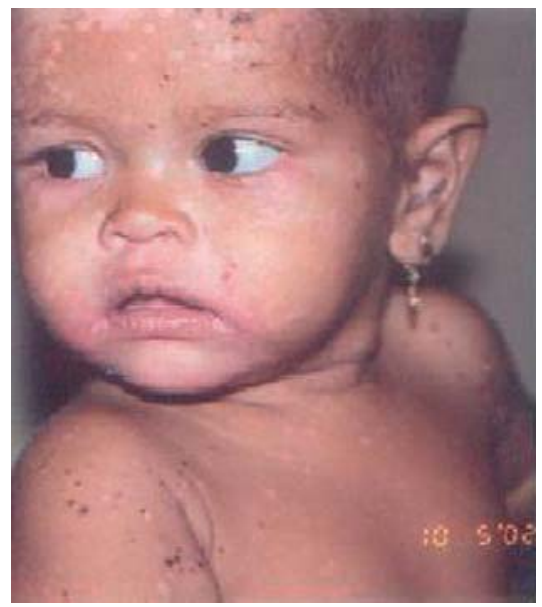

Figure 2 Complete clearing of lesions following zinc therapy.

normally present in the intestine. The clinical features usually start following weaning, when the protective effect of the zinc binding ligand from the mother's milk is no longer present. Periorificial dermatitis is the hallmark for diagnosis and the response to oral or parenteral zinc is dramatic.

A Palit, A C Inamadar Department of Dermatology, BLDEA's SBMP Medical College, Hospital \& Research Centre, Bijapur, Karnataka, India; aparuna1@rediffmail.com 